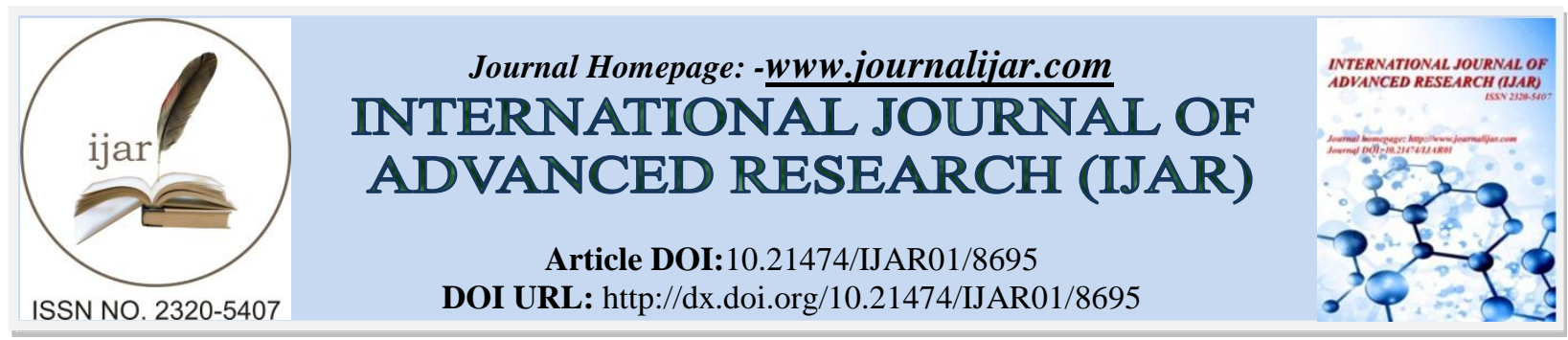

RESEARCH ARTICLE

\title{
EFFECT OF MALACHITE GREEN ON FECUNDITY AND LONGEVITY OF DROSOPHILA NASUTA ALBOMICANS.
}

Yarajarla Ramesh Babu.

Drosophila Research Laboratory, Department of Applied Genetics, Karnatak University, Dharwad - 580003, Karnataka, India.

\section{Manuscript Info}

Manuscript History

Received: 15 January 2019

Final Accepted: 17 February 2019

Published: March 2019

Key words:-

Drosophila nasuta albomicans,

Malachite green, Fecundity, fertility.

\section{Abstract}

Feeding behaviour was an important element in insects which involves the detection, the initiation of ingestion, and the consumption of food. Malachite green was an environmentally persistent and acutely toxic to a wide range of aquatic and terrestrial animals. It causes serious public health hazards and also possess potential environmental problem. Malachite green was highly cytotoxic to mammalian cells and carcinogenic to liver, thyroid and other organs of experimental animals. Incidences of tumors in lungs, breast and ovary and also been reported from rats exposed to malachite green. The larval and adult stages of Drosophila nasuta albomicans were fed in different concentrations of Malachite green. These dietary induced Malachite green showed greater influence on the fecundity of flies, and subsequently it was showed decreased fecundity and lifespan when compared to control.

Copy Right, IJAR, 2019,. All rights reserved.

\section{Introduction:-}

Dietary restriction (DR), the reduction of nutrient intake short of malnutrition, appears to improve measures of human health and extends the lifespan of various organisms ranging from yeast to primates (Fontana et al., 2010). Dietary restriction (DR) refers to a moderate reduction of food intake that leads to extension of lifespan beyond that of normal, healthy individuals. Many organisms face a challenge of meeting their optional nutritional requirement for somatic and reproductive growth under natural conditions. Nutrition plays prominent roles in aging, health, metabolism, and disease (Min and Tartar, 2006). Unlike mammals, where the foetus develops in the mother's womb, fertilized eggs of insects develop outside the female body (Bauerfeind and Fischer, 2005). All insects go through a larval phase of development that precedes metamorphosis (Awmack and Leather, 2002). Soon after hatching, larvae forage for nutrients in their vicinity and eventually find a favorable niche that contains an adequate supply of food. This phase of insect development is dedicated to feeding voraciously, and fulfils the much-needed food requirements for the subsequent non-feeding pupal phase. Ingested nutrients are stored as fat bodies (Bharucha, 2009), which serve as potential energy reservoirs for synthesis of macromolecules that are essential for cellular growth during larval and pupal stages (Holehan and Merry, 2013).

Malachite green has now become a highly controversial compound due to the risks it posses to the consumers of treated fish (Alderman and cliften Hardy,1993) includuing its genotoxic carcinogenic properties (Fernades et.al 1991,Roo, 1995).The US Food and Drug administration has nominated malachite 
green has priority chemical for carcinogenicity testing (culp and Belond,1996).There is concern about the fate of malachite green and its reduced form, Leucomalachite green in aquatic and terrestrial ecosystems since they occur as contaminats and are potential human health hazards. Bills et.al (1997) made a detail study on LC50 values of malachite green and observed the effect of $\mathrm{pH}$,temperature and exposure time on the toxicity of dye. Their study indicates that toxicity of malachite green increases with rise in temperature. It also produces chromosomal derangement in chironomid larvae and fruit fly (Drsophila melanogaster).

The present study was undertaken to ascertain the various concentrations of malachite green on fecundity and lifespan in Drosophila nasuta albomicans.

\section{Materials and Method:-}

\section{Drosophila strain, media, and culture conditions}

Drosophila nasuta albomicans stocks were maintained in an uncrowded culture condition at $22 \pm 1^{\circ} \mathrm{C}, 70 \%$ humidity and 12h: 12h light and dark cycles in standard wheat cream agar medium. From the stock the virgin females and unmated males were collected within 6 hours of eclosion and were aged for 2 days. On the third day a single virgin female and an unmated male were transferred to a fresh food media vial $(25 \times 100 \mathrm{~mm})$ and as well as different concentrations of Malachite green for egg laying. Likewise, three successive changes were made every alternate day.

\section{Fecundity assay}

Fecundity was assayed by counting number of eggs laid. Flies were successively transferred into fresh vials containing media every alternate day for 6 days. Eggs were allowed to hatch till pupation. Further, the same sets of vials were assessed for the emergence of the adult flies and likewise the fertility was recorded for the total productivity (Harini, 2011).

\section{Lifespan assay}

Synchronous cultures of 2-3-day-old files were obtained as described earlier and transferred into vials containing basal medium supplemented with experimental and control. Each group including the control had 10 vials each with 14-20 flies per vial with equal sex ratio. Flies were transferred to fresh media every third day. Dead flies were counted and removed daily throughout the experiment.

\section{Statistical Analysis:-}

Mean fecundity and lifespan were subjected to one-way ANOVA, for multiple comparisons we used Tukey's HSD by using SPSS 20.0

\section{Results:-}

Nutrients were important factors in determining the reproductive success and lifespan of flies. In the present study it was reported that control flies has enhanced the fecundity and longevity when compared with the all the other concentrations of the fed experimental diets (Malachite green) as shown in fig 1.

The analysis of variance for fecundity and lifespan showed significant values in all the concentrations. Thus the data obtained reveal significant increase in the lifespan of females compared to males fig 2. 


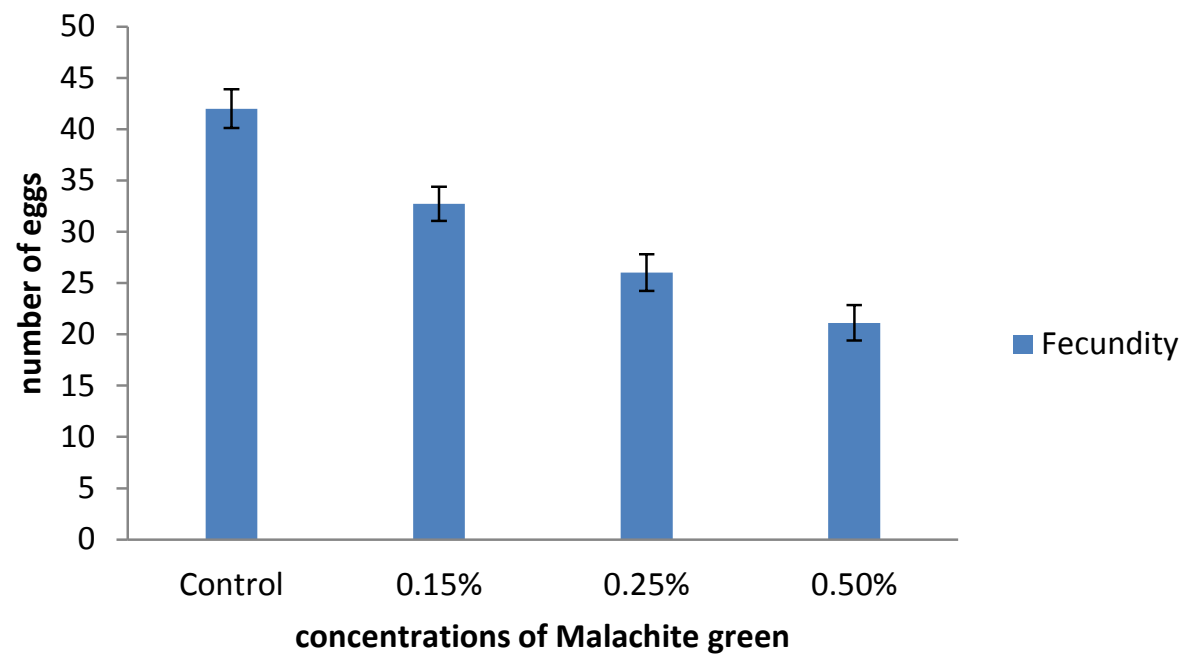

Figure 1:-Mean $( \pm$ SE) Fecundity on exposure to different concentrations of Malachite green in Drosophila nasuta albomicans.

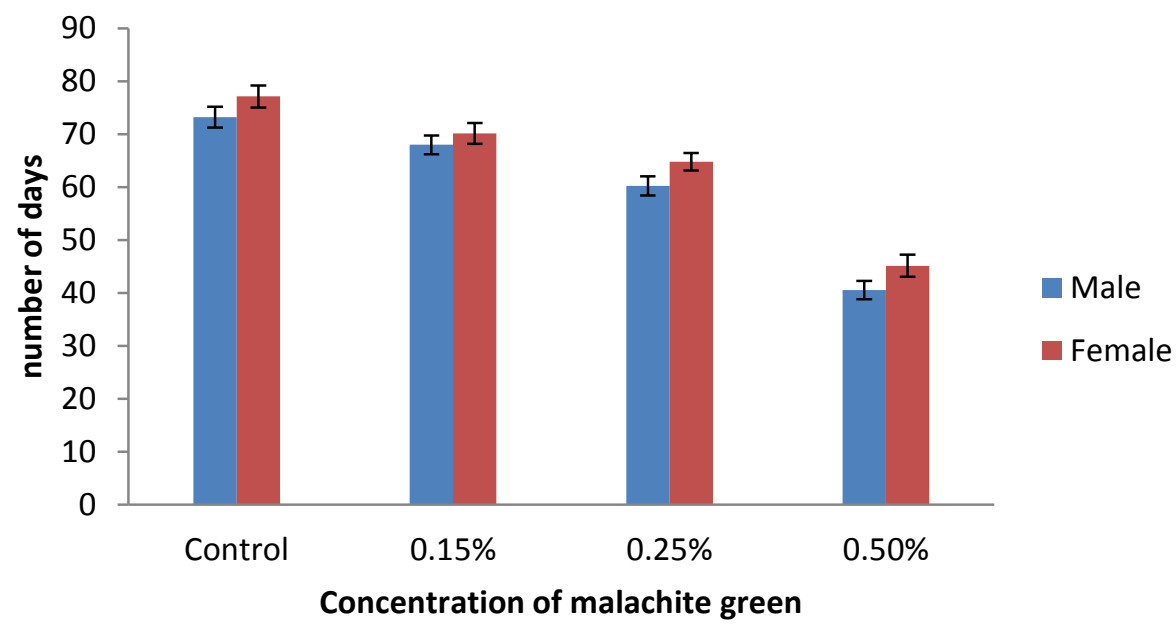

Figure 2:-Mean $( \pm \mathrm{SE})$ life span of Drosophila nasuta albomicans an exposure to different concentrations of Malachite green.

\section{Discussion:-}

Feeding was a reliable means of extending lifespan in model organisms (Weindruch and Walford, 1988). The repeated observation that dietary restriction retards aging in phylogenetically diverse species ranging from yeast to primates is the cornerstone of a fertile working hypothesis that diet regulates lifespan and aging through a universal mechanism that has been conserved throughout evolution. Commonalities between life extending mutations in several species suggest a plausible account of how genes and diet might regulate growth and aging by converging on energy and nutrient-responsive pathways. In view of this, the present study has found that the fecundity and lifespan of Drosophila can be decreased in the concentrations of malachite green.

Studies using more tractable insect models have shown that poor nutrition during development generally results in detrimental fitness effects including decreased size, fecundity, and life span (Dmitriew and Rowe, 2011). The most obvious way by which environmental variation may influence body condition and fecundity is via nutritional effects resulting from variability in food type availability. In general terms, diet effect can be classified as either quantitative (i.e., food availability) or qualitative (i.e., food composition). The quantitative effects are evident, since animals obtain energy and other nutritional requirements from food. Thus, under a natural range of conditions there is a positive correlation between food availability and body condition or fecundity. 


\section{Reference:-}

1. Alderman D.J and Clifton-Hadley R.S.(1993) Malachite green: apharmacokinetic study in rainbow trout, Oncorhynchus mykiss (Walbaum). J. Fish Dis. 16 (4), 297-311.

2. Awmack C.S and Leather S.R. (2002) Host plant quality and fecundity in herbivorous insects. Annual Review of Entomology. 47; 817-844.

3. Barnes A.I, Wigby S, Boone J.M, Partridge L and Chapman T. (2008) Feeding, fecundity and lifespan in female Drosophila melanogaster. Proc. R. Soc. 275; 1675-1683.

4. Bauerfeind S.S and Fischer K. (2005) Effects of adult-derived carbohydrates, amino acids and micronutrients on female reproduction in a fruit-feeding butterfly. Journal of Insect Physiology. 51; 545-554.

5. Bharucha K.N. (2009) The epicurean fly: using Drosophila melanogaster to study metabolism. Pediatr. Res. 65; 132-137.

6. Bills T.D, Marking L.L, Chandler J.H. (1977) Malachite green: its toxicity to aquatic organisms, persistence and removal with activated carbonInvestig. Fish Contr., 75 .

7. Carey J.R, Harshman L.G and Liedo P. (2005) Longevity-fertility trade-offs in the tephritid fruit fly, Anastrepha ludens, across dietary-restriction gradients. Aging Cell. 7; 470-477.

8. Colasurdo N, Gélinas Y and Despland E. (2009) Larval nutrition affects life history traits in a capital breeding moth.J Exp Biol. 212; 1794-1800.

9. Culp S.J and Beland F.A. (1996) Malachite green: a toxicological review. J. Am. Coll. Toxicol. 15, $219-238$.

10. Fernandes C, Lalitha V.S and Rao V.K. (1991) Enhancing effects of malachite green on the development of hepatic preneoplastic lesions induced by N-nitrosodiethylamine in rats. Carcinogenesis 12, 839-845.

11. Fontana L, Partridge L and Longo V.D. (2010) Extending healthy life span-from yeast to humans. Science. $328 ; 321-326$.

12. Grandison R.C, Piper M.D and Partridge L. (2009) Amino-acid imbalance explains extension of lifespan by dietary restriction in Drosophila. Nature.462; 1061-1064.

13. Harini B.P. (2011) Varaition in life history traits in few members of immigrans species group of Drosophila exposed to light and dark cycle. The Bioscan. 6(1); 157-162.

14. Holehan A.M and Merry B. J. (2013) The experimental manipulation of aging by diet. Biol Rev Camb Philos Soc. $61 ; 362-368$.

15. Luckinbill L.S and Clare M. (1985) Selection for lifespan in Drosophila melanogaster. Heredity. 55; 9-18.

16. Min K.J and Tatar M. (2006) Restriction of amino acids extends lifespan in Drosophila melanogaster. Mech. Ageing Dev. 127; 643-646.

17. Weindruch R and walford R.L. (1988) The retardation of aging and disease by dietary restriction. J. Gerontol. A Biol. Sci. 241; 324-328. 